\title{
Side-Detection of Out-Coupled Core Light from a Microfluidic Fiber Microslit
}

\author{
Y. Lai ${ }^{1}$, J. Petrovic ${ }^{1}$, T. Butler ${ }^{2}$, K. Sugden ${ }^{1}$, I. Bennion ${ }^{1}$ \\ ${ }^{1}$ Photonics Research Group, School of Engineering and Applied Science, Aston University, Birmingham B4 7ET, United Kingdom \\ ${ }^{2}$ FiberLogix Ltd, Watford WD18 9QP, United Kingdom \\ email:lai_yicheng@dsi.a-star.edu.sg
}

\begin{abstract}
The interactions of the core-propagating light with an intersecting microslit within a conventional single-mode fiber are investigated. Orientation-dependent out-coupling of core light was utilized to create side-detection, miniature fiber rotation sensors. (C)2008 Optical Society of America
\end{abstract}

OCIS codes: (230.4000) Microstructure fabrication; (060.2370) Fiber optics sensors

\section{Introduction}

Microfluidic fiber devices are attractive for modern biomedical, chemistry and sensing applications due to its unique functionalities and advantages over conventional-sized systems. Among various proposed device schemes, a concept to improve fluid channel integration and miniaturization by engineering microchannels into conventional fibers was recently demonstrated [1]. Such device configurations achieve effective light-fluid interactions by fabricating the microfluidic channels to intersect the fiber core, and the optical response of the fiber device is modified by the fluids within the designed microchannels. Apart from the use of standard fibers, such schemes permit channel design flexibility and offer lateral access for the fluid analyte to interact with the core-propagating light.

To date, these microfluidic fiber devices have largely relied on the responses of the core-propagating fields [2]. Depending on the channel geometry and index, one can anticipate an appreciable amount of light scattering at the channel-core interface and the detection of these light fields can convey information on the fluid parameters. Unlike the core-confined light, the scattered light fields can potentially facilitate side-detection schemes as well as provide information on the orientation of the microfluidic channel with respect to a fixed reference point outside the fiber.

In this paper, we investigate the interactions of the core-propagating light with an intersecting microslit within a conventional single-mode fiber. The numerical analysis highlighted the scattering of the core-propagating light field at the core-slit interface and, more importantly, is found to be dependent on the slit's orientation. Based on these results, a $52 \mu \mathrm{m}$-long fiber rotation sensor with a simple side-detection scheme is demonstrated experimentally.

\section{Simulation and Experiment}

In this study, we consider a microslit of dimensions $52 \mu \mathrm{m} \times 3 \mu \mathrm{m} \times 125 \mu \mathrm{m}$ (length $\times$ width $\times$ depth) that intersects the core of a standard single-mode fiber. The propagation of light in the fiber was simulated by solving 3D scalar Helmholz equation. Commercial software RSoft based on the beam propagation method (BPM) was used. Field was propagated by implicit Crank-Nicholson scheme and the nonparaxiality was resolved by the wide angle BPM that used $(1,1)$ Pade approximant. Transparent boundary conditions with radius $75 \mu \mathrm{m}$ were used to out-couple the scattered field from the simulation region. Given that the fiber device is immersed in the fluid, the index of the fiber surrounding medium and the slit were equal. Resolution of the grid was set to $0.2 \mu \mathrm{m}$ in the fiber cross section and the integration step along the fiber, $\Delta \mathrm{z}=0.05 \mu \mathrm{m}$ was refined to $0.01 \mu \mathrm{m}$ at the vicinity of core-slit interfaces. Two different fluid indices, $\mathrm{n}=1.333$ and $\mathrm{n}=1.448$, were used for computation and the field profiles within the fiber along orthogonal view planes for each case are shown in Fig. 1.
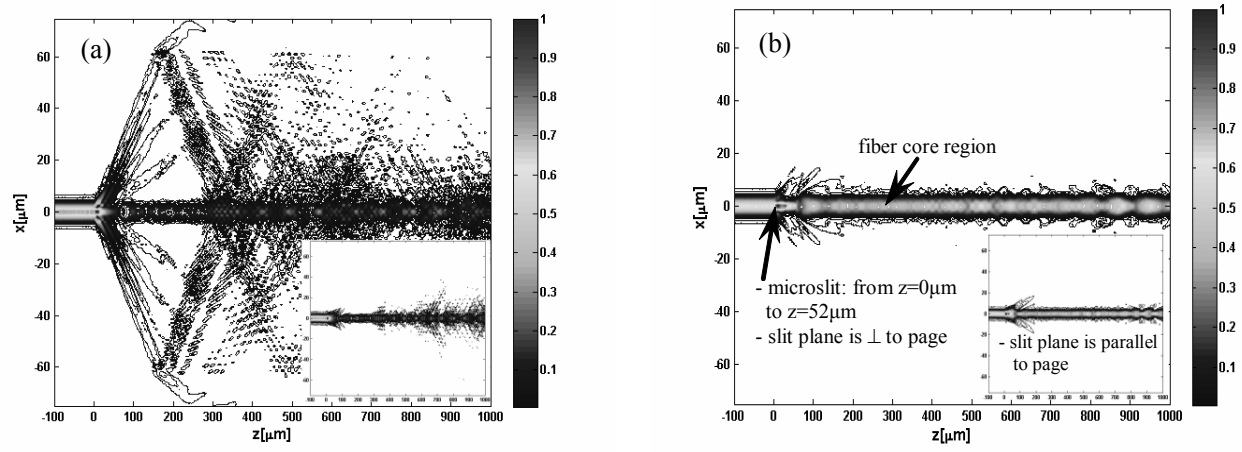

Fig. 1. Field pattern within the fiber viewed along and perpendicular (insets) to the plane of the microslit for (a) $n=1.333$ and, (b) $n=1.448$. 


\section{CFN4.pdf}

It is evident that light scattering, induced by the core-slit interface, is more extensive when $\mathrm{n}=1.333$ and the field distributions differ distinctly along orthogonal planes of view. The scattering effect is much subdued expectedly when the fluid index is closer to that of the core as shown in Fig. 1b. The interactions of the propagating light field with the slit, core and cladding interfaces lead to the resultant complex field profiles along the fiber. With the out-coupling of the scattered fields from the cladding to the surroundings, the microslit has effectively enabled out-coupling of core-propagating light into the fiber surroundings.
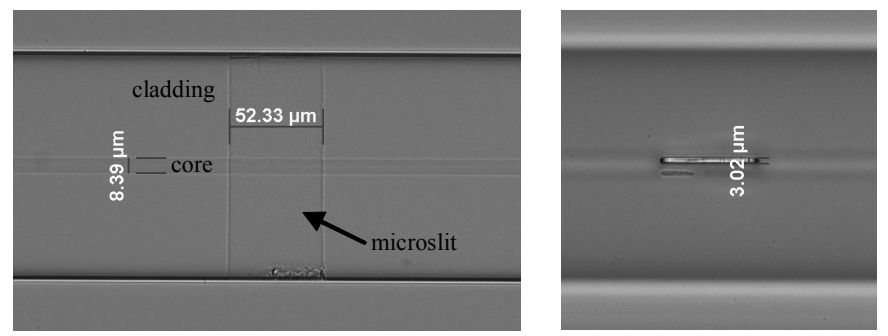

Fig. 2. Microscope images of the microslit fabricated into the fiber viewed along orthogonal planes.

In the experiment, the microslit was fabricated based on femtosecond laser exposure combined with chemical etching as detailed in [1] and the resultant structure observed under the microscope is as shown in Fig. 2. To monitor the out-coupled light from the fiber microslit device, the fiber was mounted on two rotators with the microslit centred above a photodiode (active area $1 \mathrm{~mm}^{2}$ ). Individual measurements were conducted with the microslit immersed in index fluids of indices $n=1.333$ and $n=1.448$ respectively. The glass cover over the photodiode allows the microslit to remain submerged in respective index fluids. Furthermore, care was taken to ensure no air-bubbles were trapped within the microslit. A C-band broadband light source was launched into the fiber device from one end and the amount of out-scattered light was monitored based on photocurrent detection. The microslit was rotated in steps of $30^{\circ}$ and the photocurrent readings were taken from a picoammeter. The variations of the detected photocurrent with the rotation angle are shown in Figure 3 with the data trend lines drawn in grey.
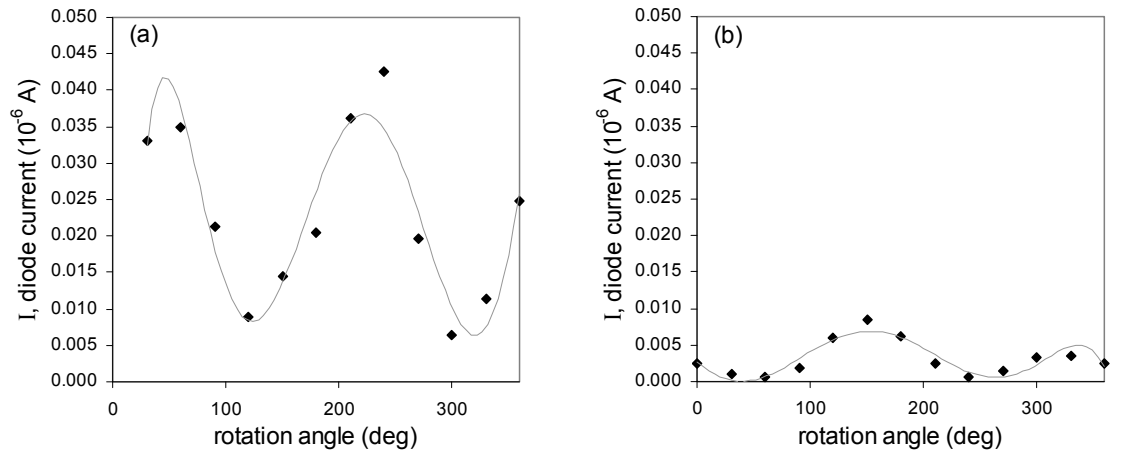

Fig. 3. Side-detection photodiode current versus rotation angle of the microslit when (a) $n=1.333$ and, (b) $n=1.448$.

The oscillatory variations of the photocurrent with rotation angle clearly illustrate that the out-coupled light is dependent on the microslit's orientation. The detection extinction ratio $\left(I_{\max }-I_{\min }\right)$ evidently depends on the slit index, which further conforms to the numerical analysis predictions. It is also of interest to note that by monitoring the transmission spectrum of the microslit device, and given that the scattering loss is the main mechanism of the insertion loss of the device, the spectral range of the out-coupled light is found to extend over the entire C-band.

\section{Conclusion}

The interactions of the core-propagating light with an intersecting microslit within a single-mode fiber have been studied. Guided mode out-coupling is observed due to the scattering effect induced by the microslit. The outcoupled light exhibits orientation dependency which allows a miniature, wide spectral bandwidth, side-detection fiber rotation sensor to be realized. The results highlight a potential new attribute of such microfluidic fiber devices for photonics applications.

\section{References}

[1] Y. Lai et al., "Microchannels in conventional single-mode fibers", Opt. Lett., 31, 2559-2561, 2006.

[2] K. Zhou et al., "Refractometer based on micro-tunnel in fiber Bragg grating by chemically assisted femtosecond laser processing", Opt. Exp., $15,15848-15853,2007$ 